\title{
Klippel-Trenaunay Syndrome, a Rare Cause of Hematochezia
}

\author{
Eric Omar Then a, d, Andrew Ofosu ${ }^{\mathrm{b}}$, Prashanth Rawla ${ }^{\mathrm{c}}$, \\ Tagore Sunkara ${ }^{\mathrm{b}}$, Vinaya Gaduputia
}

\begin{abstract}
Klippel-Trenaunay syndrome (KTS) is a rare disorder with a wide array of clinical manifestations. It is primarily a disorder of vascular malformations that is classically associated with the development of hemangiomas and venous malformations. Rarely will KTS present with visceral involvement. Venous malformations of the gastrointestinal tract in specific are an exceedingly rare manifestation of KTS. When present, it can result in life-threatening bleeding that is oftentimes difficult to manage. Our case highlights a 53-year-old woman who presented to our clinic with chronic rectal bleeding secondary to KTS.
\end{abstract}

Keywords: Hematochezia; Rectal bleeding; Klippel-Trenaunay syndrome; Anemia; Hemangioma

\section{Introduction}

Klippel-Trenaunay syndrome (KTS) is a rare disorder resulting from vascular malformations, which was first described by two French physicians in 1900 [1]. It is characterized by a triad of hemangiomas (secondary to capillary dysplasias), soft tissue or bone hypertrophy, and venous anomalies [2]. Its pathogenesis remains unclear, but recent studies have suggested a mutation of the PIK3CA gene to be responsible [3]. The physiology behind visceral hemangiomas is thought to occur from shunting of blood in venous malformations of affected limbs into the internal iliac vein. Subsequently, the poor drainage of vesical, genital and rectal veins results in dilated venous mal-

Manuscript submitted September 18, 2018, accepted October 8, 2018

aDivision of Gastroenterology and Hepatology, SBH Health System, 4422 Third Ave, Bronx, NY 10457, USA

bDivision of Gastroenterology and Hepatology, The Brooklyn Hospital Center, Clinical Affiliate of The Mount Sinai Hospital, 121 Dekalb Ave, Brooklyn, NY 11201, USA

cDepartment of Internal Medicine, Memorial Hospital of Martinsville and Henry County, 320 Hospital Drive, Martinsville, VA 24115, USA

${ }^{\mathrm{d}}$ Corresponding Author: Eric Omar Then, 4422 Third Avenue, Bronx, NY 10457, USA. Email: ericomarthen27@yahoo.com

doi: https://doi.org/10.14740/gr1092 formations in their respective locations [4].

\section{Case Report}

Our patient is a 53-year-old woman with a medical history significant for KTS that was referred to our gastroenterology clinic from her hematologist for rectal bleeding. The patient was known to our institution and had been receiving intravenous iron treatment for chronic iron deficiency anemia. The patient's anemia was secondary to known bladder and colon hemangiomas, which resulted in chronic hematuria and hematochezia. This, coupled with chronic right lower extremity varicosities, was enough to make the presumptive diagnosis of KTS. The patient reported first noticing the rectal bleeding 13 years prior, occurring intermittently with bowel movement. Despite her symptoms, the patient had persistently refused colonoscopy until presenting to our clinic. Notable laboratory workup included hemoglobin of $11 \mathrm{~g} / \mathrm{dL}$, hematocrit of $35.5 \%$, a mean corpuscular volume of $79 \mathrm{fL}$ and iron studies within normal limits. Physical exam was notable for right lower extremity varicose veins and a soft non-tender mass over the lumbar spine. After appropriate counseling, the patient agreed to undergo colonoscopy. Colonoscopy was done and revealed a tortuous colon with a purplish hue (Fig. 1). This was accompanied by enlarged submucosal blebs in the distal segment (sigmoid to rectum) (Fig. 2). There were also punctate erythematous mucosa within the same segment and internal hemorrhoids with stigmata of recent bleeding. Unfortunately, there were no lesions amenable to argon plasma coagulation, cautery or hemostatic clipping at the time. The patient followed up 4 weeks later with persistence of hematochezia, but with no hemodynamic instability.

\section{Discussion}

Present day, KTS has an estimated annual incidence rate of less than $1 \%$, specifically occurring in every 1 in 100,000 live births. It has not been shown to have a predilection for race or gender, making its diagnosis yet more difficult for the clinician to elucidate [5]. In addition, its symptoms may mimic those of other disorders. These include, in particular, Parkes-Weber syndrome (PWS) and Proteus syndrome [6]. The prior will cause arteriovenous malformations, while the later has clas- 

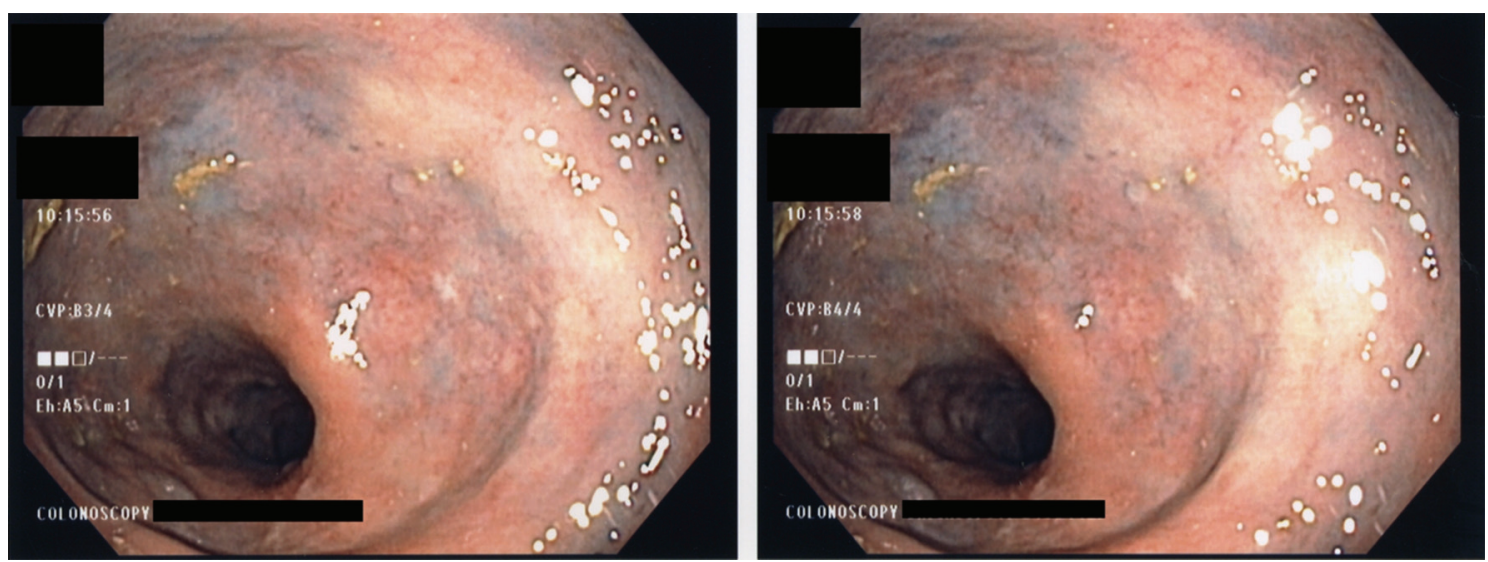

Figure 1. Colonoscopy showing purplish discoloration of the entire mucosa.

sically been associated with soft tissue overgrowth. Both of these symptoms are well documented to occur in KTS. The hallmark feature of PWS that distinguishes it from KTS, is the presence of arteriovenous fistulas [7]. Proteus syndrome, on the other hand, will present with verrucous nevi and subcutaneous lipomas, which are absent in KTS [8]. It is important to make the distinction between these disorders due to the mortality associated with KTS-induced gastrointestinal bleeding.

The presentation of KTS has a wide spectrum with the ability to affect every organ system. This is due to the unpredictable nature of vascular malformations [6]. Gastrointestinal KTS is a rare occurrence. Life-threatening gastrointestinal hemorrhage due to diffuse cavernous hemangiomas has been reported in up to $12.5 \%$ of patients afflicted with KTS [9]. Superimposed bladder hemorrhage has been reported to occur in $1 \%$ of these cases [10]. The most common reported sites of gastrointestinal bleeding are the distal colon and rectum but can occur along the entire gastrointestinal tract [11]. Further,
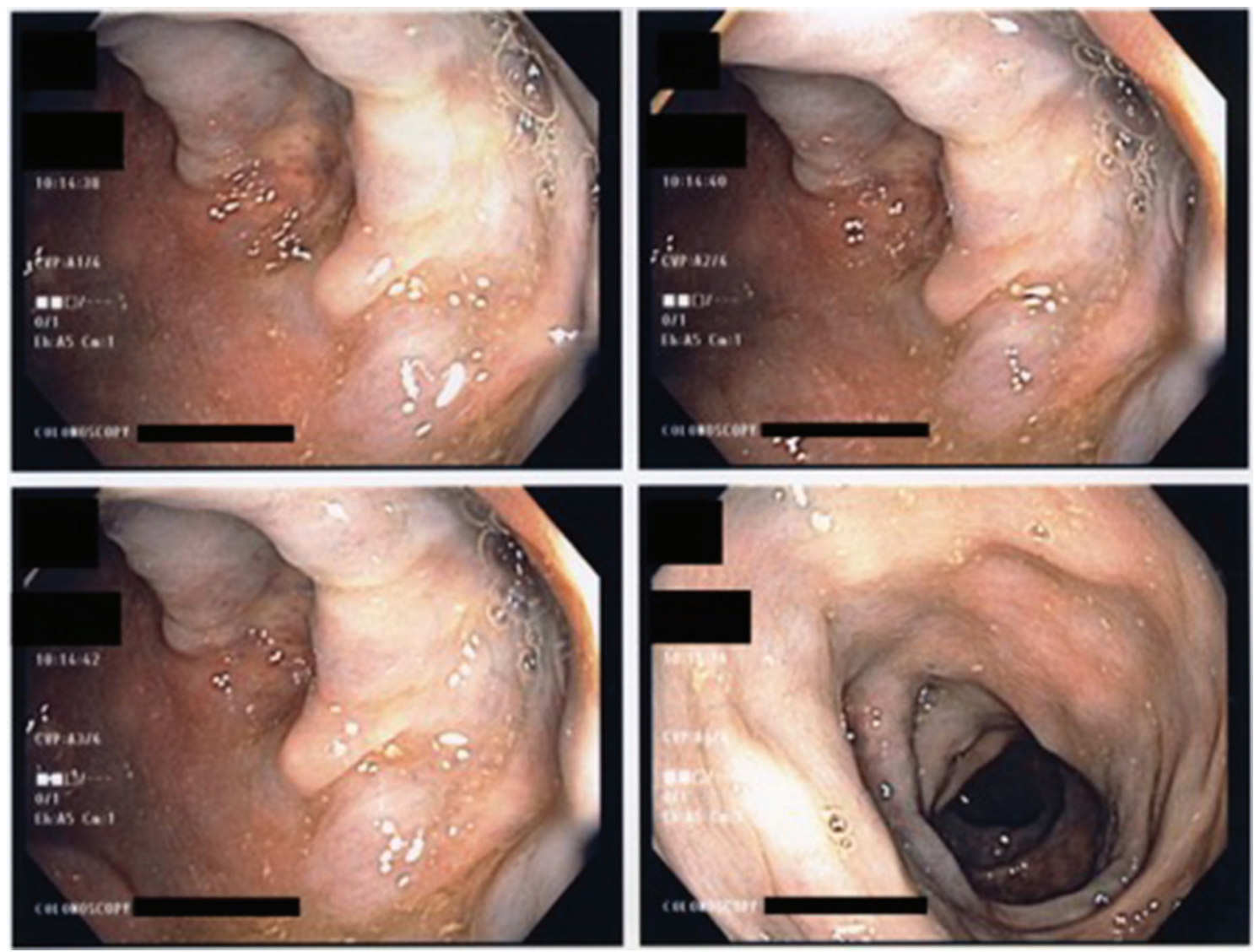

Figure 2. Colonoscopy revealing diffuse venous congestion resulting in submucosal blebs. 
KTS has also been associated with Kasabach-Merritt phenomenon in which platelet sequestration with secondary consumption of clotting factors occurs within the venous sinusoids of the vascular malformation [12]. Our patient was spared from this phenomenon, but did present with significant bleeding from the sigmoid colon, rectum and bladder.

Diagnosis of gastrointestinal KTS is most readily accomplished through esophagogastroduodenoscopy (EGD) or colonoscopy. Capsule endoscopy has also been shown to be an effective tool in identifying gastrointestinal KTS [13]. Endoscopic ultrasound (EUS) is a newer modality that in addition to diagnosis can also make the distinction between KTS and portal hypertension in patients with underlying cirrhosis [14]. Endoscopic findings characteristic of KTS include bluish submucosal angiomatous lesions accompanied by dilated submucosal vascular structures $[15,16]$. Biopsies are not routinely taken as it may precipitate massive bleeding which further increases mortality in patients with pre-existent hematochezia [17].

Treatment of gastrointestinal KTS is centered on conservative measures. Due to the high risk of bleeding, endoscopic interventions are seldom pursued. Mild cases can be treated with simple iron replacement, while more severe cases necessitate blood transfusions. In cases of transfusion dependency, surgical resection or angiography with embolization has been shown to be effective alternatives [18]. In our patient, iron replacement was successful in maintaining adequate levels of hemoglobin, in lieu of chronic gastrointestinal bleeding.

\section{Conclusions}

In conclusion, gastrointestinal KTS is a rare cause of bleeding that can be life-threatening. Given its wide range of manifestations, it can be a challenge to diagnose. When suspected, a prompt investigation should take place with EGD or colonoscopy. This allows the clinician the ability to rule out other causes of massive gastrointestinal hemorrhage that are amenable to intervention. Once diagnosed, gastrointestinal KTS should be treated conservatively. More severe cases should be treated with surgical resection or embolization.

\section{Conflict of Interest}

None of the authors have any financial conflicts of interest.

\section{Disclosure}

None.

\section{Author Contributions}

All authors have confirmed that the article is not under consideration for review at any other journal. All authors have made contributions to the article and have reviewed it before submission.

\section{Financial Support}

No funding to disclose.

\section{References}

1. Klippel M, Trenaunay P. Du noevus variqueux osteohypertrophique. Arch Gen Med. 1900;185:641-672.

2. Wang ZK, Wang FY, Zhu RM, Liu J. Klippel-Trenaunay syndrome with gastrointestinal bleeding, splenic hemangiomas and left inferior vena cava. World J Gastroenterol. 2010;16(12):1548-1552.

3. Vahidnezhad H, Youssefian L, Uitto J. Klippel-Trenaunay syndrome belongs to the PIK3CA-related overgrowth spectrum (PROS). Exp Dermatol. 2016;25(1):17-19.

4. Rotholtz N, Bun ME, Laporte M, Sandra L, Carlos P, Mezzadri N. Rectal bleeding in Klippel-Trenaunay syndrome: treatment with laparoscopic ultralow anterior resection with intersphincteric dissection. Surg Laparosc Endosc Percutan Tech. 2009;19(5):e206-209.

5. Gizzi G, Fabbri C, Fuccio L. A 28-year-old woman with hypertrophic right leg and haematochezia. Gut. 2015;64(10):1538.

6. Sreekar H, Dawre S, Petkar KS, Shetty RB, Lamba S, Naik S, Gupta AK. Diverse manifestations and management options in Klippel-Trenaunay syndrome: a single centre 10-year experience. J Plast Surg Hand Surg. 2013;47(4):303-307.

7. Ziyeh S, Spreer J, Rossler J, Strecker R, Hochmuth A, Schumacher M, Klisch J. Parkes Weber or Klippel-Trenaunay syndrome? Non-invasive diagnosis with MR projection angiography. Eur Radiol. 2004;14(11):2025-2029.

8. Puri KJPS, Malhotra SK, Jain A. Klippel Trenaunay and Proteus Syndrome overlap - a diagnostic dilemma. Egyp Derm Onl J. 2009;5(2):10.

9. Thosani N, Ghouri Y, Shah S, Reddy S, Arora G, Scott LD. Life-threatening gastrointestinal bleeding in Klippel-Trenaunay syndrome. Endoscopy. 2013;45(Suppl 2 UCTN):E206.

10. Kocaman O, Alponat A, Aygun C, Gurbuz Y, Sarisoy HT, Celebi A, Senturk O, et al. Lower gastrointestinal bleeding, hematuria and splenic hemangiomas in KlippelTrenaunay syndrome: a case report and literature review. Turk J Gastroenterol. 2009;20(1):62-66.

11. Li T, Hu SY, Chen ZT, Chen ZQ, Zhi XT. Colorectal cavernous hemangioma in Klippel-Trenaunay syndrome: a rare cause of abdominal pain and hematochezia. Surgery. 2015;157(2):402-404.

12. Garg PK, Kumar A. Unusual cause of rectal bleeding. Klippel-Trenaunay syndrome (KTS). Saudi J Gastroenterol. 2011;17(6):421-422.

13. Weersma RK, Thijs WJ, Vosmaer GD, Koornstra JJ. Capsule endoscopy in Klippel-Trenaunay syndrome. Endoscopy. 2007;39(Suppl 1):E58.

14. Vazquez-Sequeiros E, Sorbi D, Kamath PS, Wiersema MJ. Klippel-Trenaunay-Weber syndrome: role of EUS. Gastrointest Endosc. 2001;54(5):660-661. 
15. Samo S, Sherid M, Husein H, Sulaiman S, Yungbluth M, Vainder JA. Klippel-Trenaunay syndrome causing lifethreatening GI bleeding: a case report and review of the literature. Case Rep Gastrointest Med. 2013;2013:813653.

16. Arguedas MR, Shore G, Wilcox CM. Congenital vascular lesions of the gastrointestinal tract: blue rubber bleb nevus and Klippel-Trenaunay syndromes. South Med J.
2001;94(4):405-410.

17. Deepinder F. GI bleeding, colon varicosities, and visceral enlargement as a manifestation of Klippel-Trenaunay syndrome. Clin Gastroenterol Hepatol. 2011;9(12):e126-127.

18. Tetangco EP, Arshad HM, Silva R. Klippel-Trenaunay Syndrome of the rectosigmoid colon presenting as severe anemia. ACG Case Rep J. 2016;3(4):e161. 